\title{
Essai de corrélation entre les caractéristiques du climat, de la végétation et des sols au Burkina Faso
}

\author{
François Jean Pascal PALLO * et Norbert SAWADOGO \\ Institut de l'Environnement et de Recherches Agricoles (INERA)-04 BP 8645 Ouagadougou 04- Burkina Faso. \\ *Auteur correspondant, E-mail: jeanpallo@ hotmail.com; Tel. 226-76-63-92-81
}

\section{RESUME}

L'étude a été réalisée en vue d'appréhender les relations entre le climat, la végétation et les sols au Burkina Faso. Pour cela, six sites regroupant 84 points d'observation ont été considérés. Ils sont répartis le long d'un gradient climatique allant de la zone sub-soudanienne à la zone sahélienne. Au niveau de chaque site, cinq variables climatiques ont été retenues. A chaque point d'observation, cinq caractéristiques du couvert végétal, douze propriétés physico-chimiques et biologiques de l'horizon supérieur des sols ont été déterminées. L'application du test de corrélation de rang de Spearman entre les différents paramètres révèle que la péjoration du climat de la zone humide à la zone sèche se traduit, au niveau des strates arborées et arbustives par un appauvrissement de la biodiversité végétale. Par ailleurs, la diminution de la pluviosité moyenne annuelle dans les zones nord soudanienne et sub-sahélienne entraîne un ralentissement des processus biologiques de décomposition de la matière organique des sols ; ce qui conduit à une augmentation des teneurs en carbone total et en azote total des sols. L'étude a mis aussi en évidence l'importance des facteurs stationnels sur les caractéristiques de la végétation et de la matière organique. Elle suggère alors, la prise en compte de l'ensemble de ces facteurs dans l'élaboration des projets de lutte contre la désertification.

(C) 2010 International Formulae Group. All rights reserved.

Mots clés: Matière organique, activité biologique, strates arborées, herbacées

\section{INTRODUCTION}

La littérature abonde d'écrits relatant les effets du climat sur les sols. Ils relèvent qu'à travers la température et la pluviosité, le climat est le facteur déterminant des processus de pédogenèse (Duchaufour, 1977). Il influence également les caractéristiques de la matière organique et l'activité biologique des sols (Kirschbaum, 1995 ; Leiros et al., 1999 ; Franzluebbers, 2001). Si bien qu'à l'échelle planétaire, la carte des sols coïncide avec celle du climat. Parallèlement, aux principales zones climatiques correspondent des formations végétales spécifiques qui se distinguent les unes des autres autant par leur densité, leur structure que par leur composition floristique. Ainsi, les variations latitudinales du climat s'accompagnent de celles de la végétation et des sols. Au Burkina Faso, chaque facteur pris isolément, a fait l'objet de nombreux travaux. En effet, les données climatiques du pays sont 
régulièrement recueillies par la direction de la météorologie nationale à partir de dix stations synoptiques et analysées pour appréhender les phénomènes de changements climatiques. Concernant la végétation, plusieurs études dont celles portant sur les pâturages (Toutain et de Wispeleare, 1978 ; Grouzis et Sicot, 1983), sur l'élevage et les potentialités pastorales sahéliennes (IEMVT/CTA, 1987) ont été réalisées. Le programme de recherche intégré sur la lutte contre l'aridité dans l'Oudalan a aussi permis d'étudier les caractéristiques de la végétation et du cycle de l'eau dans cette province septentrionale du pays (Sicot, 1978 ; le Grand, 1979 ; Grouzis, 1979). Les résultats de plusieurs travaux (Guinko, 1984 ; Zoungrana, 1992 ; Thiombiano, 2005), auxquels s'ajoutent ceux obtenus par l'Université Agronomique de Wageningen (UAW, 1991) dans le cadre de l'aménagement et de la gestion des terroirs sylvo-pastoraux au sahel et par les universités et structures nationales de recherches forestières, contribuent à accroître les connaissances sur ce facteur écologique. Quant aux données pédologiques, elles proviennent pour l'essentiel de la carte de reconnaissance des sols du Burkina Faso au 1/500 0000 (5 feuilles) réalisée en 1969 par l'Institut de Recherche pour le Développement (IRD) et des travaux effectués par le Bureau National des Sols depuis sa création en 1974 et par diverses structures (Bunasols, 1989a). A l'échelle nationale, une correspondance a aussi été établie entre la distribution des trois grands types de végétation (steppes, savanes, forêts) et le découpage des zones climatiques (MEE, 1996). En conséquence, la présente étude vise à préciser les caractéristiques de la végétation qui changent avec le climat et à montrer que ces modifications dépendent également des propriétés des sols. En effet, dans le système d'évaluation des terres pour la foresterie (FAO, 1984), les conditions générales du climat (radiation solaire, température, humidité) et les qualités des sols telles que la disponibilité en oxygène, en éléments nutritifs, les conditions d'enracinement etc. sont des exigences pour le développement des espèces forestières. Les travaux ont été réalisés sur six sites situés dans des formations naturelles de longue durée (forêts classées, réserves de terres, parcelles mises en défens) protégées contre les feux de brousse, le pâturage, la coupe de bois et autres activités anthropiques (p.e. agriculture). Ils sont placés le long d'un gradient climatique qui va de la région la plus humide à celle qui est la plus sèche et traverse les principales unités de sol du pays.

\section{MATERIEL ET METHODES}

\section{Cadre géographique}

Les travaux se sont déroulés en Afrique de l'ouest, au Burkina Faso (Figure 1). Les sites sont localisés suivant un gradient climatique allant de la forêt classée de Niangoloko (ouest- sud) aux aires protégées de Sambonaye/Dori (nord-est). Au plan climatique et au niveau des territoires phytogéographiques (Tableau 1), les sites se scindent en deux groupes (Guinko, 1984). Le premier rassemble Niangoloko, Farakoba, Tiogo, Sâ. Il est situé dans la zone et le territoire soudaniens. Yabo et Sambonaye constituent le second groupe. Ils se trouvent dans la partie sahélienne du Burkina Faso. Le pédoclimat est ustique, isohyperthermique de Niangoloko à Yabo, aridique et hyperthermique à Sambonaye (Boyadgiev, 1980). Le substratum géologique est dominé par les formations du précambrien $\mathrm{D}$ et $\mathrm{C}$ à Niangoloko, Tiogo, Yabo, Sambonaye ; par celles du précambrien A et du tertiaire respectivement à Farakoba et Sâ (Hottin et Ouédraogo, 1976). Les sols des différents sites correspondent dans la base de référence mondiale pour les ressources en sols (FAO, 1998), aux Lixisols à Tiogo et Yabo ; aux 
Cambisols, à Sâ et Sambonaye. Ce dernier site renferme également des Luvisols. Les Arénosols et les Ferralsols constituent les groupes de référence respectifs de Niangoloko et de Farakoba.

\section{Caractérisation du climat}

Pour caractériser le climat de chaque site, cinq paramètres ont été considérés : la pluviosité moyenne annuelle ( $\mathrm{P}$ en $\mathrm{mm})$, la température moyenne annuelle $\left(\mathrm{T}^{\circ} \mathrm{C}\right)$, l'évapo-transpiration potentielle (ETP en $\mathrm{mm}$ ), les ratios $\mathrm{P} / \mathrm{ETP}$ et $\mathrm{P} / \mathrm{T}$ (facteur de pluie). Les valeurs moyennes de ces variables ont été calculées pour la période 1982-2000.

\section{Caractérisation pédologique}

Chaque site a fait l'objet d'une prospection pédologique détaillée à l'échelle de 1/10000è. Conformément à la méthodologie élaborée par le Bureau National des sols (Bunasols, 1989b), la méthode de quadrillage systématique a été appliquée. La description des profils de sol dans les fosses s'est effectuée selon les directives FAO en la matière (FAO, 1994); suivie d'un prélèvement des échantillons de sol, horizon par horizon, pour analyse au laboratoire. En fonction de la superficie de chaque site, le nombre de profils de sol décrit s'élève à 14 à Niangoloko; 12 à Farakoba; 16 à Tiogo, Sâ, et Yabo ; 10 à Sambonaye. Les travaux se sont déroulés en septembre-octobre, période correspondant à la fin de la saison pluvieuse. Les caractères morphologiques et les propriétés physico-chimiques des sols de ces différents sites sont rapportés par Pallo (2009). Les propriétés édaphiques considérées ici, ne concernent que l'horizon A humifère des profils de sol. Son épaisseur varie selon les sites et les types de sol. Celle-ci est en moyenne de $15 \mathrm{~cm}$ pour les sites de Niangoloko, Farakoba, Tiogo, Yabo, et Sambonaye et de $26 \mathrm{~cm}$ pour le site de Sâ.
Trois groupes de propriétés ayant une influence sur les conditions d'enracinement et de développement des végétaux, d'après le système d'évaluation des terres pour la foresterie (FAO 1984), ont été retenus :

- les propriétés physiques: granulométrie/ texture

- les propriétés chimiques : carbone et azote totaux

- les paramètres biologiques : respiration du sol ( $\mathrm{C}-\mathrm{CO}_{2}$ dégagé), taux de carbone associé à la biomasse microbienne.

La granulométrie a été obtenue par la méthode de pipetage à la pipette Robinson. Les méthodes de Walkley et Black (1934) et de Keldjahl (Bremner, 1965) ont été utilisées pour le dosage respectif du carbone (C) total et de l'azote $(\mathrm{N})$ total. Pour quantifier le gaz carbonique $\left(\mathrm{CO}_{2}\right)$ dégagé, la méthode de Dommergues (1960) a été utilisée. La détermination du carbone de la biomasse microbienne (CBM) a été réalisée selon la méthode de Jenkinson et Powlson (1976) à partir d'échantillons de sol fumigés et non fumigés.

Le taux de minéralisation globale du carbone (TMG) a été calculé suivant la formule ci-après:

TMG = [quantité du carbone dégagée/quantité de carbone du sol] x 100

\section{Caractérisation de la végétation.}

La végétation a été caractérisée lors de la prospection pédologique des sites. Afin de prendre en compte le maximum d'espèces végétales ayant une influence sur le profil de sol considéré, la végétation qui se développe dans un rayon de $5 \mathrm{~m}$ autour de ce dernier a été décrite conformément aux méthodes de la FAO en la matière (FAO, 1994) et à la classification des formations végétales élaborée par le service de l'aménagement forestier (Bunasols, 1989b). La caractérisation de la végétation a permis à partir des paramètres de hauteur et de recouvrement des 
plants, l'identification des classes et sous classes de la formation végétale de chaque point d'observation. Elle a été suivie du recensement des espèces végétales constituant chacune des strates arborée, arbustive, herbacée. Au niveau des deux premières strates, le nombre de pieds par espèce a été également déterminé. Par point d'observation, les caractéristiques ainsi retenues ont été les suivantes :

-Pour la strate arbustive (L): le nombre de pieds (Li), le nombre d'espèces végétales (Le) et Le nombre de familles (Lf) auxquelles appartiennent ces espèces.

-Pour la strate herbacée $(\mathrm{H})$ : Le nombre d'espèces végétales (He), Le nombre de familles (Hf) auxquelles appartiennent ces espèces.

\section{Traitement statistique des données}

Les degrés de liaison entre les différents paramètres indiqués ci- dessus ont été appréhendés en appliquant le test de Spearman (1904). Les coefficients de corrélation de rang (rs) ont été calculés en utilisant le logiciel Minitab pour Window version 13-31 et appréciés aux probabilités suivantes : $\mathrm{p}<0,05$ (significatif : *); $\mathrm{p}<0,01$ (hautement significatif : **); $\mathrm{p}<0,001$ (très hautement significatif : ***).

\section{RESULTATS}

Des différentes caractéristiques du climat, consignées au Tableau 2, on note que de la zone subsoudanienne à la zone sahélienne, la pluviosité moyenne annuelle (P) et ses dérivés (P/T et P/ETP) diminuent; tandis que la température moyenne annuelle (T) augmente. Une corrélation négative significative a été établie entre $\mathrm{T}$ et les précédents paramètres ( $\left.\mathrm{rs}=-0,89^{*}\right)$. L'évapotranspiration potentielle (ETP) varie en dents de scie et n'est corrélée avec aucune variable climatique.
Concernant la végétation (Tableau 3), les nombres moyens de pieds (Li), d'espèces (Le) et de famille (Lf) de la strate arborée/arbustive, par point d'observation décroissent de Niangoloko à Sambonaye. La strate herbacée des sites de la zone nord soudanienne (Tiogo et Sâ) possède en moyenne le moins d'espèces et de familles. La caractéristique $\mathrm{Li}$ est respectivement corrélée avec Le ( $\left.\mathrm{rs}=0,83^{*}\right)$ et $\mathrm{Lf}\left(\mathrm{rs}=0,83^{*}\right)$. La corrélation entre $\mathrm{Le}$ et $\mathrm{Li}$ est positive très hautement significative ( $\mathrm{rs}=1,00 * * *)$.

Au plan pédologique (Tableau 4), les sols de Niangoloko sont ceux qui renferment le moins de particules fines $(\varnothing<50 \mu \mathrm{m})$ et ont, en conséquence, le rapport sables/argiles, le plus élevé. Le phénomène inverse est observé à Sâ. Les sols des sites localisés dans la zone nord soudanienne et sub-sahélienne (Yabo) détiennent les plus forts taux de carbone et d'azote totaux. Au niveau des paramètres biologiques, les résultats indiquent que les deux paramètres que sont la respiration du sol et le taux de carbone associé à la biomasse microbienne, varient en dents de scie tout au long du gradient climatique considéré. Leurs valeurs dans les sols du sahel (Sambonaye) sont supérieures à celles des sols subsoudaniens (Niangoloko).Les résultats du test de Spearman ont révélé une corrélation positive entre le taux de particules fines $(\mathrm{A}+\mathrm{LT})$ et la teneur en carbone total $\left(r s=0,84^{*}\right)$. Il en est de même entre le taux de particules fines et les teneurs en azote total $(\mathrm{rs}=0,89 *)$. Le taux de particules fines $(\mathrm{A}+\mathrm{LT})$ est aussi positivement corrélé avec le taux de carbone associé à la biomasse microbienne $\left(\mathrm{rs}=0,83^{*}\right)$. Avec le rapport sables/argiles, ces coefficients respectifs deviennent négatifs. Par ailleurs, des coefficients de corrélation de rang positifs, hautement significatifs ( $\mathrm{rs}=0,94 * *$ ) ont été obtenus entre chacun des paramètres liés à la pluviométrie (P, P/T, P/ETP) d'une part et Le, Lf d'autre part. La température moyenne annuelle (T) et le taux 
F. J. P. PALLO et al. / Int. J. Biol. Chem. Sci. 4(5): 1839-1850, 2010

Tableau 1 : Données générales sur les milieux d'étude.

\begin{tabular}{|c|c|c|c|c|c|c|}
\hline Sites/n nordre & Niangoloko/ 1 & Farakoba/ 2 & Tiogo/ 3 & Sâ/ 4 & Yabo/ 5 & Sambonaye/ 6 \\
\hline Coordonnées & $10^{\circ} 16^{\prime} 20^{\prime \prime} \mathrm{N}$ & $11^{\circ} 10^{\prime} 54^{\prime \prime} \mathrm{N}$ & $12^{\circ} 13^{\prime} 38^{\prime \prime} \mathrm{N}$ & $12^{\circ} 39^{\prime} 20^{\prime \prime} \mathrm{N}$ & $13^{\circ} 1^{\prime} 55^{\prime \prime} \mathrm{N}$ & $14^{\circ} 8^{\prime} 40^{\prime \prime} \mathrm{N}$ \\
\hline géographiques & $4^{\circ} 53^{\prime} 27^{\prime \prime} \mathrm{W}$ & $4^{\circ} 16^{\prime} 21^{\prime \prime} \mathrm{W}$ & 2०49’6’’W & $3^{\circ} 17^{\prime} 53^{\prime \prime} \mathrm{W}$ & $1^{\circ} 32^{\prime} 43^{\prime \prime} \mathrm{W}$ & 0³'10’’E \\
\hline Climat & subsoudanien & sud-soudanien & nord-soudanien & nord-soudanien & subsahélien & sahélien \\
\hline $\begin{array}{l}\text { Régime } \\
\text { hydrique des sols }\end{array}$ & Ustique & Ustique & Ustique & Ustique & Ustique & Aridique \\
\hline $\begin{array}{l}\text { Régime } \\
\text { thermique des sols }\end{array}$ & Isohyperthermique & Isohyperthermique & Isohyperthermique & Isohyperthermique & Isohyperthermique & Hyperthermique \\
\hline $\begin{array}{l}\text { Territoire } \\
\text { phytogéographique }\end{array}$ & $\begin{array}{c}\text { secteur } \\
\text { soudanien méridional } \\
\text { (district de la Comoé) }\end{array}$ & $\begin{array}{c}\text { secteur } \\
\text { soudanien méridional } \\
\text { (district ouest } \\
\text { Mouhoun) }\end{array}$ & $\begin{array}{l}\text { secteur } \\
\text { soudanien } \\
\text { méridional } \\
\text { (district est } \\
\text { Mouhoun) } \\
\end{array}$ & $\begin{array}{c}\text { secteur } \\
\text { soudanien } \\
\text { septentrional }\end{array}$ & secteur subsahélien & $\begin{array}{c}\text { secteur } \\
\text { sahélien strict }\end{array}$ \\
\hline $\begin{array}{l}\text { Substratum } \\
\text { géologique }\end{array}$ & $\begin{array}{c}\text { +migmatites et } \\
\text { granites } \\
\text { indifférenciés } \\
\text { + roches volcano } \\
\text { sédimentaires et } \\
\text { métavolcanites }\end{array}$ & $\begin{array}{c}\text { +formations } \\
\text { sédimentaires } \\
\text { à dominante gréseuse }\end{array}$ & $\begin{array}{c}\text { +migmatites et } \\
\text { granites } \\
\text { indifférenciés } \\
\text { + roches volcano } \\
\text { sédimentaires et } \\
\text { méta volcanites }\end{array}$ & $\begin{array}{c}\text { Continental } \\
\text { terminal }\end{array}$ & $\begin{array}{c}\text { +migmatites et granites } \\
\text { indifférenciés } \\
+ \text { roches volcano sédimentaires } \\
\text { et métavolcanites }\end{array}$ & $\begin{array}{c}\text { +migmatites } \\
\text { leptynites et } \\
\text { granites } \\
\text { indifférenciés } \\
\text { + roches volcano } \\
\text { sédimentaires ; } \\
\text { tark waïen }\end{array}$ \\
\hline $\begin{array}{l}\text { Sols } \\
\text { (groupe de } \\
\text { référence) }\end{array}$ & Arenosols & Ferralsols & Lixisols & Cambisols & Lixisols & $\begin{array}{l}\text { + Cambisols } \\
\text { + Luvisols }\end{array}$ \\
\hline
\end{tabular}


F. J. P. PALLO et al. / Int. J. Biol. Chem. Sci. 4(5): 1839-1850, 2010

Tableau 2 : Caractéristiques climatiques des sites étudiés.

\begin{tabular}{|c|c|c|c|c|c|c|}
\hline Site & Niangoloko & Farakoba & Tiogo & Sà & Yabo & Sambonaye \\
\hline Pluviosité moyenne annuelle $(\mathrm{P})$ en mm & 1020,7 & 1015,8 & 797,5 & 755,3 & 707,8 & 442,6 \\
\hline $\begin{array}{l}\text { Evapotranspiration potentielle moyenne } \\
\text { annuelle (ETP) en mm }\end{array}$ & 2011,1 & 2011,1 & 1923,1 & 2444,0 & 2091,8 & 2027,0 \\
\hline Température moyenne annuelle $(\mathrm{T}){ }^{\circ} \mathrm{C}$ & 27,4 & 27,3 & 28,1 & 28,7 & 28,5 & 29,7 \\
\hline P/ETP & 0,507 & 37,20 & 28,38 & 26,31 & 24,83 & 14,90 \\
\hline Facteur d'aridité : $\mathrm{P} / \mathrm{T}$ & 37,25 & 37,20 & 28,38 & 26,31 & 24,83 & 14,90 \\
\hline
\end{tabular}

Tableau 3 : Caractéristiques du couvert végétal par profil de sol.

\begin{tabular}{|c|c|c|c|c|c|c|}
\hline Site/profils de sol & & Niangoloko/14 & Farakoba/12 & Tiogo/16 & Sâ/16 & Yabo/16 \\
\hline \multirow{3}{*}{ Strate arborée/arbustive (L) } & $\mathrm{Li}$ & $199,86 \pm 70,39$ & $121,08 \pm 76,59$ & $36,93 \pm 19,10$ & $53,25 \pm 24,43$ & $68,8 / \pm 49,00$ \\
\hline & Le & $24,07 \pm 6,33$ & $20,50 \pm 5,56$ & $9,62 \pm 3,03$ & $7,18 \pm 1,56$ & $9,06 \pm 2,32$ \\
\hline & Lf & $15,7 / \pm 2,67$ & $14,00 \pm 2,89$ & $6,06 \pm 1,81$ & $5,25 \pm 1,34$ & $5,37 \pm 1,71$ \\
\hline \multirow{2}{*}{ Strate herbacée $(\mathrm{H})$} & $\mathrm{He}$ & $4,64 \pm 1,64$ & $7,58 \pm 1,44$ & $3,62 \pm 1,50$ & $3,87 \pm 1,58$ & $5,87 \pm 2,06$ \\
\hline & $\mathrm{Hf}$ & $3,71 \pm 1,54$ & $2,90 \pm 0,99$ & $1,68 \pm 0,60$ & $2,68 \pm 0,87$ & $4,31 \pm 1,54$ \\
\hline
\end{tabular}


F. J. P. PALLO et al. / Int. J. Biol. Chem. Sci. 4(5): 1839-1850, 2010

Tableau 4 : Caractéristiques pédologiques et biologiques de l'horizon supérieur des sols des sites étudiés.

\begin{tabular}{|c|c|c|c|c|c|c|c|}
\hline \multicolumn{2}{|c|}{ Site /profil de sol } & \multirow{2}{*}{$\begin{array}{l}\text { Niangoloko /14 } \\
2,21 \pm 1,53\end{array}$} & \multirow{2}{*}{$\begin{array}{l}\text { Farakoba/12 } \\
7,75 \pm 3,45\end{array}$} & \multirow{2}{*}{$\frac{\text { Tiogo/16 }}{9,31 \pm 2,62}$} & \multirow{2}{*}{$\begin{array}{l}\text { Sâ /16 } \\
54,45 \pm 15,52\end{array}$} & \multirow{2}{*}{$\frac{\text { Yabo /16 }}{14,0 \pm 2,88}$} & \multirow{2}{*}{$\begin{array}{l}\text { Sambonaye/10 } \\
21,75 \pm 8,82\end{array}$} \\
\hline & Argiles \% & & & & & & \\
\hline$\cdot \stackrel{\vec{\pi}}{\vec{\pi}}$ & $\mathrm{A}+\mathrm{LT} \%$ & $11,95 \pm 2,86$ & $24,78 \pm 3,72$ & $28,85 \pm 8,30$ & $87,42 \pm 4,65$ & $62,18 \pm 1,85$ & $32,63 \pm 9,16$ \\
\hline$\sum^{\pi}$ & Sables/argiles & $50,51 \pm 17,74$ & $11,55 \pm 5,08$ & $8,31 \pm 2,64$ & $0,43 \pm 0,83$ & $2,81 \pm 0,60$ & $3,68 \pm 1,78$ \\
\hline \multirow{3}{*}{$\stackrel{0}{\Sigma}$} & $\mathrm{C}$ total $\mathrm{mgkg}^{-1}$ sol & $3,39 \pm 1,13$ & $3,78 \pm 0,74$ & $7,53 \pm 2,52$ & $8,71 \pm 2,34$ & $10,26 \pm 2,29$ & $3,78 \pm 1,60$ \\
\hline & $\mathrm{N}$ total $\mathrm{mgkg}^{-1}$ sol & $0,35 \pm 0,06$ & $0,31 \pm 0,03$ & $0,47 \pm 0,16$ & $0,88 \pm 0,21$ & $0,77 \pm 0,13$ & $0,36 \pm 0,13$ \\
\hline & $\mathrm{C} / \mathrm{N}$ & $9,53 \pm 2,20$ & $12,28 \pm 2,04$ & $15,90 \pm 1,35$ & $10,15 \pm 1,189$ & $13,16 \pm 1,64$ & $10,52 \pm 1,23$ \\
\hline \multirow{6}{*}{ 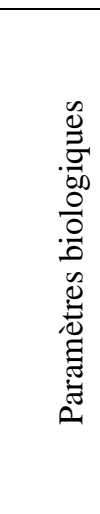 } & $\begin{array}{l}\text { C. } \mathrm{CO}_{2} \text { dégagé } \\
1^{\text {er }} \text { jour } \mathrm{mgC} / 100 \mathrm{~g}\end{array}$ & $0,70 \pm 0,18$ & $2,38 \pm 0,35$ & $1,77 \pm 0,79$ & $1,56 \pm 0,42$ & $3,32 \pm 0,64$ & $3,34 \pm 1,26$ \\
\hline & $\begin{array}{l}\text { Cumul C. } \mathrm{CO}_{2} \text { dégagé } \\
31^{\text {ème }} \mathrm{jr} \\
\mathrm{mgC} / 100 \mathrm{~g}\end{array}$ & $11,9 \pm 3,01$ & $21,43 \pm 6,09$ & $12,20 \pm 5,91$ & $11,76 \pm 4,74$ & $29,88 \pm 6,81$ & $15,87 \pm 8,32$ \\
\hline & TMG $1^{\mathrm{er}}$ jour $\%$ & $0,22 \pm 0,06$ & $0,64 \pm 0,12$ & $0,22 \pm 0,06$ & $0,18 \pm 0,06$ & $0,32 \pm 0,07$ & $0,98 \pm 0,48$ \\
\hline & TMG $31^{\text {ème }}$ jour $\%$ & $3,69 \pm 0,91$ & $5,72 \pm 1,65$ & $1,57 \pm 0,35$ & $1,35 \pm 0,44$ & $2,91 \pm 0,55$ & $4,38 \pm 1,95$ \\
\hline & $\mathrm{CBM}$ en $\mathrm{mgC} / 100 \mathrm{~g}$ & $12,24 \pm 2,24$ & $7,70 \pm 3,32$ & $15,18 \pm 5,82$ & $15,92 \pm 5,15$ & $20,42 \pm 5,08$ & $13,08 \pm 3,20$ \\
\hline & $\begin{array}{l}\text { CBM en pourcent de } \\
\text { C. total }\end{array}$ & $3,87 \pm 1,08$ & $2,04 \pm 0,81$ & $2,02 \pm 0,44$ & $1,89 \pm 0,67$ & $2,05 \pm 0,39$ & $3,84 \pm 1,41$ \\
\hline
\end{tabular}

Signification des abréviations : $\mathrm{MO}=$ Matière organique ; A+LT \% $=$ argiles + limons totaux \%;C $=$ Carbone ; $\bar{N}=$ azote $; \mathrm{C} / \mathrm{N}=$ rapport carbone $/$ azote ;

$\mathrm{CBM}=$ teneur en carbone de la biomasse microbienne $; \mathrm{TMG}=$ Taux de minéralisation globale du carbone. 


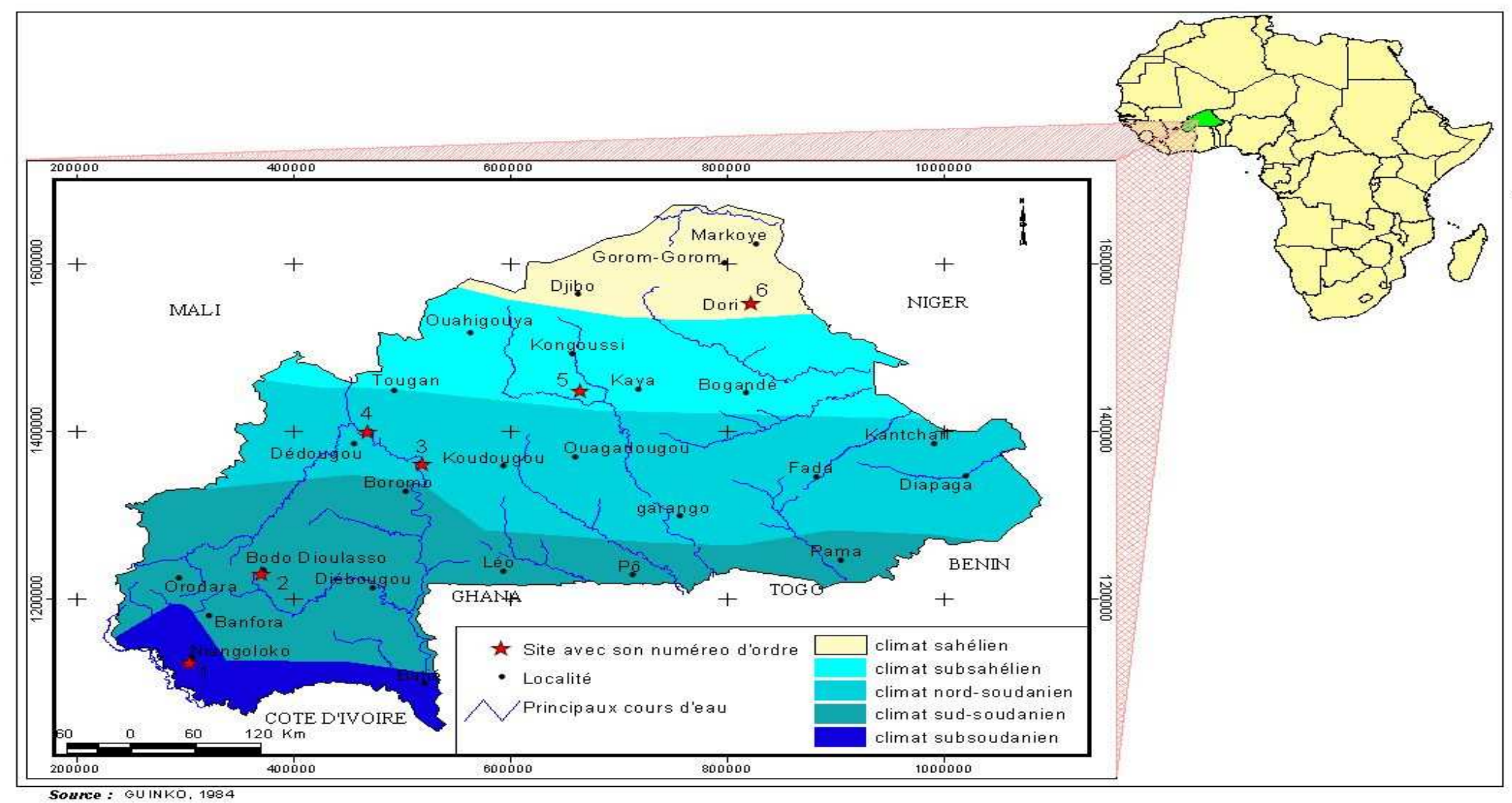

Figure 1 : Localisation des sites 
d'argiles (A) sont négativement corrélés avec ces caractéristiques de la strate arborée/arbustive. Le et Lf sont positivement corrélées avec le rapport sables/argiles $\left(\mathrm{rs}=0,83^{*}\right)$. Le taux moyen de minéralisation globale du carbone (TMG) du premier jour et son cumul au 31è jour d'incubation ont chacun une corrélation positive avec le nombre moyen d'espèces végétales de la strate herbacée; rs s'élevant respectivement à $0,84 *$ et à $0,89 *$.

\section{DISCUSSION}

L'appréciation qui se dégage des résultats présentés ci-dessus est que la zone climatique sub-soudanienne comporte des formations végétales dont les strates arborée et arbustive sont plus riches en espèces que les autres zones climatiques étudiées. Cela est imputable plus aux précipitations relativement abondantes qu'elle reçoit qu'aux températures moyennes annuelles plus douces qui y règnent. En ce sens, c'est la pluviosité moyenne annuelle et ses dérivés (P/T, P/ETP) qui contrôlent davantage la production primaire nette de ces strates comme observé ailleurs par Parton et al. (1987) et par Burke et al. (1989).

En remontant vers le nord, on note tout au long du gradient climatique considéré un appauvrissement de la biodiversité spécifique végétale se traduisant par une diminution du nombre de pieds, d'espèces et de familles. A Sâ, la réduction de la densité des strates arborée et arbustive peut être aussi attribuée aux mauvaises conditions d'enracinement provoquées par les fortes teneurs en particules argilo-limoneuses (Bunasols, 1990). Sur ce site, les teneurs élevées en carbone total des sols sont imputables aux fortes quantités de ces particules fines. Par les liens que ces particules contractent avec les fractions organiques, elles assurent une protection physique contre les attaques biologiques (Buyanovsky et al., 1994). Les relations entre le taux d'azote total et celui des particules argilo-limoneuses résultent de cette protection. En effet, les microorganismes sont responsables pour 80 à $90 \%$ de la minéralisation de la matière organique des sols (Lavelle et al., 1993). Par ailleurs, de la zone subsoudanienne (Niangoloko) à la zone subsahélienne (Yabo), la diminution de la pluviosité moyenne annuelle s'accompagne d'une augmentation des teneurs en carbone et azote totaux. Inversement, l'accroissement de la température moyenne annuelle se traduit par des taux plus élevés de $\mathrm{C}$. total et $\mathrm{N}$. total. Ces résultats sont contraires à ceux obtenus dans d'autres régions du monde (Burke et al., 1989; Amelung et al., 1997; Alvarez et Lavedo, 1998). En effet, selon les auteurs précités, la vitesse de décomposition de la matière organique des sols (MOS) est accrue avec l'augmentation de la température moyenne annuelle. Cependant, nos observations se justifient. Elles sont dues à la réduction de la durée de la saison des pluies qui passe de 7-8 mois à Niangoloko, à moins de 5 mois à Yabo; si bien que la matière organique a tendance à s'accumuler dans les sols de ce site, plus par manque d'humidité pour la poursuite des processus biologiques de minéralisation du carbone que pour des raisons thermiques. En outre, le régime hydrique de ces sols est plus ustique que celui des sols de Niangoloko et de Farakoba (Boyadgiev, 1980). Quant à la diminution du taux de carbone associé à la biomasse microbienne (CBM) lorsque la pluviosité moyenne augmente, elle résulte du fait que ce paramètre biologique est corrélé avec la quantité de carbone total. Outre la mise en évidence de corrélations entre les caractéristiques du climat, du couvert végétal et de la MOS, l'étude illustre aussi l'importance de la fraction minérale du sol sur le développement de la végétation. Ainsi, l'augmentation du rapport sables/argiles s'accompagne de celle du nombre de pieds 
(Li), d'espèces (Le) et de familles (Lf). La raison est que des valeurs élevées de cette variable participent à l'amélioration des conditions d'enracinement des sols.

\section{Conclusion}

Il ressort de toutes ces données qu'au Burkina Faso, la péjoration du climat de la zone subsoudanienne à la zone sahélienne entraîne au niveau des strates arborée et arbustive, une réduction de la densité, du nombre d'espèces végétales et de familles auxquelles elles appartiennent. Les faibles pluviosités moyennes annuelles alliées à des conditions pédoclimatiques peu favorables aux processus biologiques de minéralisation $\mathrm{du}$ carbone favorisent l'accumulation de C total dans l'horizon supérieur des sols des zones climatiques nord soudanienne et subsahélienne. L'étude a cependant, mis en évidence, l'influence du matériau (texture) sur le couvert végétal, sur les caractères généraux de la matière organique et sur l'activité biologique des sols; si bien que les résultats obtenus émanent des interactions climatfacteurs stationnels. Elle a enfin montré que même de faibles variations latitudinales de la température moyenne annuelle ont des incidences considérables sur la végétation et l'épi- pédon. Les résultats renforcent l'idée que les activités exécutées dans le cadre de la lutte contre la désertification doivent prendre en considération les facteurs écologiques tels que le climat, la végétation et le sol et les relations qui existent entre ces derniers, pour notamment mieux réussir les opérations de reboisement.

\section{REFERENCES}

Alvarez R, Lavedo RS. 1998. Climate, organic matter and clay content relationships in the Pampa and chaco soils, Argentina. Geoderma, 83: 127-141.

Amelung W, Flach KW, Zech W. 1997. Climatic effects on soil organic matter composition in the great plains. Soil Sci. Soc. Am. J., 61: 115-123.

Boyadgiev TG. 1980. Création d'un service des sols. Haute-Volta. Etat des connaissances des sols. AG : DP/UPV/74/007. Rapport technique 1. PNUD/FAO. Rome, 33p.

Bremner JM. 1965. Total nitrogen. In Method of Analysis (Part 2). Blak CA (ed). Am. Soc. Agron., 9. Madison : WI. ; 11491178.

Bunasols. 1989a. Liste des études pédologiques réalisées au Burkina Faso depuis 1955. Doc. Techn. $\mathrm{N}^{\circ} 4$, Bunasols, Ouagadougou, 56 p.

Bunasols. 1989b. Méthodes de prospection pédologique. Doc. Techn. $\mathrm{N}^{\circ}$ 5, Bunasols, Ouagadougou, $121 \mathrm{p}$.

Bunasols. 1990. Manuel pour l'évaluation des terres. Doc. Techn. $\mathrm{N}^{\circ} 6$, Bunasols, Ouagadougou, $181 \mathrm{p}$.

Burke CI, Yonker CM, Parton WJ, Cole CV, Flach K. Schimel DS. 1989. Texture, climate and cultivation effects on soil organic matter in US grassland soils. Soil Sci. Soc. Am. J., 53: 800-805.

Buyanovsky GA, Aslam M, Wagner GH. 1994. Carbon turnover in soil physical fractions. Soil Sci. Soc. Am. J., 58: 449457.

Dommergues Y. 1960. La notion de coefficient de minéralisation du carbone dans les sols. L'Agronomie Tropicale, 15(1): 55-60.

FAO (Foods and Agriculture Organization ) 1984. Land evaluation for forestry. FAO Forestry paper $\mathrm{N}^{\circ} 48$, FAO Rome, Italy, $163 \mathrm{p}$.

FAO. 1994. Directives pour la Description des Sols. 3è édition révisée. FAO: Rome, Italy; $73 \mathrm{p}$.

FAO. 1998. World Reference Base for Soils Resources. FAO: Rome, Italy; $91 \mathrm{p}$.

Franzluebbers AJ, Haney RL, Honeycutt CW, Arshad MA, Schombery HH, Hons FM. 
2001. Climatic influence on active fractions of soil organic matter. Soil Biol. Biochem., 33: 1103-1111.

Grouzis M. 1979. Structure, composition floristique et dynamique de la production de matière sèche des formations végétales sahéliennes (mare d'Oursi-Haute-Volta). ACC Lutte Contre l'Aridité dans l'Oudalan. Centre ORSTOM : Ouagadougou, Burkina Faso, 56 p.

Grouzis M, Sicot AM. 1983. Production Séquentielle du Bassin Versant de la mare d'Oursi. Application à l'estimation des potentialités pastorales. Atelier FAOISRA. Dakar 16-18/11/ 1983. Centre ORSTOM. Ouagadougou, Burkina Faso, $6 \mathrm{p}$.

Guinko S. 1984. Végétation de la HauteVolta. Thèse Doctorat es Sciences nat., Univ. Bordeaux III, Tome I. 318 p.

Hottin G, Ouédraogo OF. 1976. Carte géologique au 1/1000 000è de la république de Haute-Volta. DGM HauteVolta.

IEMVT/CTA (Institut d'Elevage et de Médecine Vétérinaire des Pays Tropicaux/Centre Technique de Coopération Agricole Rurale). 1987. Elevage et potentialités pastorales sahélienne. Synthèses cartographiques. Burkina Faso, IEMVT- Maison-Alfort, France.

Jenkinson DS, Powlson DS. 1976. The effects of biocidal treatments on metabolism in soil. V. A method for measuring soil biomass. Soil Biol. Biochem., 8(3): 209213.

Kirschbaum Muf. 1995. The temperature dependence of soil organic matter decomposition and effect of global warming on soil organic C storage. Soil Biol. Biochem., 27: 753-760.

Lavelle P, Blanchard E, Martin A. 1993. A hierarchical model for decomposition in terrestrial ecosystems: Application to soils of the humid tropics. Biotropica, 23: 130-150.

Le Grand Ph. 1979. Biomasse racinaire de la strate herbacée des formations sahélienne (étude préliminaire). ACC Lutte contre l'Aridité dans l'Oudalan. Centre ORSTOM : Ouagadougou ; 6 p.

Leiros MC, Trasar Cepeda C, Soane S, GilSotres F. 1999. Dependence of mineralization of soil organic matter on temperature and moisture. Soil Biol. Biochem., 31: 327-335.

MEE (Ministère de l'Environnement et de l'Eau) 1996. Plan d'action national pour l'environnement. Programme national d'aménagement des forêts. Ouagadougou. $62 \mathrm{p}$.

Pallo FJP. 2009. Statut de la matière organique des sols sous formations naturelles de longue durée au Burkina Faso. Thèse de Doctorat. d'état es Sciences nat. Univ. Polytechnique de Bobo Dioulasso (Burkina Faso). Vol. 1 et $2,326 \mathrm{p}$.

Parton WJ, Scimel DC, Cole CV, Ojima DS. 1987. Analysis of factors controlling soil organic matter level in great plains grassland. Soil Sci. Soc. Am. J., 51: 11731179.

Sicot AM. 1977. Cycle de l'eau et bilan hydrique dans les écosystèmes types du bassin versant de la mare d'Oursi (HauteVolta). Analyse préliminaire des données recueillies en 1977. Critiques des principes de base du bilan hydrique en milieu sahélien. ACC Lutte contre l'Aridité dans l'Oudalan. Centre ORSTOM : Ouagadougou ; 208 p.

Spearman C. 1904. The proof and measurement of association between two things. Am. J. Psych., 15: 72-101.

Thiombiano A. 2005. Les combrétacées du Burkina Faso: Taxonomie, écologie, dynamique et régénération des espèces. Thèse Doctorat d'état es Sciences 
naturelles, Univ. de Ouagadougou, Burkina Faso, 290 p

Toutain B, de Wispeleare G. 1978. Pâturage de l'ORD du Sahel et de la zone de délestage au nord-est de Fada N'Gourma. Etude agrostologique $\mathrm{N}^{\circ}$ 51. IEMVT. Maison - Alfort: France (3 tomes); $1200 p$.

UAW (Université Agronomique de Wageningen) 1991. Aménagement et gestion des terroirs sylvo-pastoraux au Sahel. Programme de la recherche
« sahel »1991-1996. Wageningen, PaysBas, 85 p.

Walkley A, Black IA. 1934. An examination of Degtjareff method for determining soil organic matter and a proposed modification of the chromic acid titration method. Soil Sci., 37: 29-38.

Zoungrana I. 1992. Recherches sur les aires pâturées au Burkina Faso. Thèse Doctorat d'état es Sciences naturelles. Univ. Bordeaux III, 227p. 